\title{
Estimating mother-to-child HIV transmission rates in Cameroon in 2011: a computer simulation approach
}

Hermine L. Nguena Nguefack ${ }^{1 *}$, Henri Gwet ${ }^{1}$, Sophie Desmonde ${ }^{2,3}$, Odile Ouwe Missi Oukem-Boyer ${ }^{4,5}$, Céline Nkenfou ${ }^{4}$, Mathurin Téjiokem6 ${ }^{6}$ Patrice Tchendjou ${ }^{6}$, Irénée Domkam ${ }^{4}$ Valériane Leroy ${ }^{2,3,7}$, Ahmadou Alioum ${ }^{2,3}$ and for the Inserm U897 Modeling Infectious Diseases in Low-Income Countries Study Group

\begin{abstract}
Background: Despite the progress in the Prevention of the Mother-to-Child Transmission of HIV (PMTCT), the paediatric HIV epidemic remains worrying in Cameroon. HIV prevalence rate for the population of pregnant women was 7.6 \% in 2010 in Cameroon. The extent of the paediatric HIV epidemic is needed to inform policymakers. We developed a stochastic simulation model to estimate the number of new paediatric HIV infections through MTCT based on the observed uptake of services during the different steps of the PMTCT cascade in Cameroon in 2011. Different levels of PMTCT uptake was also assessed.

Methods: A discrete events computer simulation-based approach with stochastic structure was proposed to generate a cohort of pregnant women followed-up until 6 weeks post-partum, and optionally until complete breastfeeding cessation in both prevalent and incident lactating HIV-infected women. The different parameters of the simulation model were fixed using data sources available from the 2011 national registry surveys, and from external cohorts in Cameroon. Different PMTCT coverages were simulated to assess their impact on MTCT. Available data show a low coverage of PMTCT services in Cameroon in 2011.

Results: Based on a simulation approach on a population of 995, 533 pregnant women, the overall residual MTCT rate in 2011 was estimated to be $22.1 \%$ (95 \% Cl: $18.6 \%-25.2 \%$ ), the 6-week perinatal MTCT rate among prevalent HIV-infected mothers at delivery is estimated at $12.1 \%$ (95\% Cl: $8.1 \%-15.1 \%)$, with an additional postnatal MTCT rate estimated at $13.3 \%$ (95\% Cl: $9.3 \%-17.8 \%$ ). The MTCT rate among children whose mothers seroconverted during breastfeeding was estimated at $20.8 \%$ (95 \% Cl: $14.1 \%-26.9 \%)$. Overall, we estimated the number of new HIV infections in children in Cameroon to be 10, 403 (95\% Cl: 9, 054-13, 345) in 2011. When PMTCT uptake have been fixed at $100 \%, 90 \%$ and $80 \%$, global MTCT rate failed to $0.9 \%$ (95 \% Cl: $0.5 \%-1.7 \%$ ), $2.0 \%$ (95 \% Cl: $0.9 \%-3.2 \%)$ and $4.3 \%$ (95\% Cl: $2.4 \%-6.7 \%)$ respectively.

Conclusions: This model is helpful to provide MTCT estimates to guide the national HIV policy in Cameroon. Increasing supply and uptake of PMTCT services among prevalent HIV infected pregnant women, as well as HIVprevention interventions including the offer and acceptance of HIV testing and counselling in lactating women could reduce significantly the residual HIV MTCT in Cameroon. A public health effort should be made to encourage health care workers and pregnant women to use PMTCT services until complete breastfeeding cessation.
\end{abstract}

Keywords: HIV, Mother-to-child transmission, Africa, Children, Breastfeeding, Modelling

\footnotetext{
* Correspondence: hermine.nguena@gmail.com

${ }^{1}$ National Advanced School of Engineering, The University of Yaoundé I, PO

Box 8390, Yaoundé, Cameroon

Full list of author information is available at the end of the article
} 


\section{Background}

It is estimated that $90 \%$ of HIV infections in children are resulting from mother-to-child-transmission (MTCT) [1]. By the end of 2011, of the 34 million adults infected with HIV worldwide, 16 million were women [2]; in subSaharan Africa, prevalence of HIV among pregnant women is high. In the absence of any intervention to prevent MTCT (PMTCT), the MTCT rate varies between $13 \%$ and $48 \%$ [1-3]. Maternal combination antiretroviral therapy (ART) together with postnatal interventions have demonstrated their efficacy in reducing substantially the risk of MTCT in African breastfed children to less than $5 \%[4,5]$. The World Health Organization (WHO) has called for the "virtual elimination" of pediatric HIV [6, 7] with the most efficient and cost-effective ways to tackle paediatric HIV being to reduce MTCT worldwide [8]. However, access to ART and the uptake of PMTCT programs remain limited and children continue to be HIVinfected [1].

In Cameroon, the prevalence of HIV was estimated to be $4.3 \%$ in the general population; a sero-surveillance survey among pregnant women showed an HIV prevalence of $7.6 \%$ in 2010 [9]. As a result the number of new paediatric infections continues to grow in Cameroon and there are still thousands of new infections each year [10]. In 2011, the UNAIDS launched the Global Plan towards the elimination of new HIV infections among children and keeping their mothers alive [7] making Cameroon, which overall MTCT risk was reported to be around 24\% [7], one of the 21 priority countries. Since 2011, Cameroon has tripled its coverage of PMTCT prophylaxis, ranging from $6.9 \%$ to $36.5 \%$ in 2011, leading to $30 \%$ fewer new HIV infections among children [11]. In 2011, Cameroon opted for the WHO Option A regimen for PMTCT prophylaxis. Continuing access of pregnant women living with HIV to prenatal HIV services and increasing access to HIV treatment for eligible children and pregnant women will reduce maternal and child mortality [11]. Cameroon has focused on strengthening PMTCT services and caring of paediatric HIV cases for the 2011-2015 period: $99.4 \%$ of health districts are equipped to provide HIV treatment services for pregnant women and children living with HIV in 2011. However, even where the most effective PMTCT interventions are available, many women and infants are lost at different steps of the PMTCT cascade [12] and the low cumulative uptake of PMTCT services does not allow controlling the extent of MTCT in Cameroon.

Clinical trials and cohort studies provide essential information in assessing PMTCT interventions; however these studies are also very time and resource-consuming, difficult to implement and not representative. Alternatively, simulation models can integrate available data and help to project long-term outcomes. To date, several published analyses have reported on simulation models to estimate MTCT of HIV. Vieira et al. developed a discrete-event, three-phase simulation, built in Visual Basic, with a stochastic semi-Markov structure to model different intervention strategies at any time, including short-course antiretroviral drugs and cessation of breastfeeding in Botswana [13]. Ciaranello et al. used local trial and programmatic data to simulate a cohort of HIV-infected, pregnant/breastfeeding women in Zimbabwe and to compare five PMTCT regimens at a fixed level of PMTCT medication uptake. The authors linked published computer simulation models to project clinical outcomes of five PMTCT strategies [14]. Another simulation model for MTCT is the Spectrum software package, developed by Stover et al. [15-17]. The Spectrum program is used to forecast key HIV prevalence and PMTCT rates based on national HIV surveillance and surveys, program statistics and epidemic patterns. To the best of our knowledge, to date there are no published models that simulate individually a cohort of pregnant women in Cameroon able to estimate MTCT rates accounting for both prevalent (infected at delivery) and incident (infected in the lactating period) maternal HIV infections. Thus, we developed a stochastic simulation model to estimate the MTCT risk (perinatal and postnatal) and the number of new paediatric infections based on the observed uptake of PMTCT programmes at different stages of the PMTCT cascade in Cameroon in 2011. PMTCT uptake is an important determinant of MTCT, we thus assessed the impact of increase of PMTCT uptake at $100 \%, 90 \%$ and $80 \%$ on the MTCT of HIV in Cameroon.

\section{Methods}

We developed a discrete event computer simulation-based approach with a stochastic structure to generate a hypothetical cohort of pregnant women followed-up through different states during pregnancy until 6-weeks postnatally (perinatal transmission, which refers to all infections detected prior to 6 weeks postpartum), and optionally until complete weaning (prevalent and incident postnatal transmission). Briefly there were 3 main health states: No HIV infection, HIV-infection, and death. The HIV-infection state were then subdivided into two sub-states :chronic HIV infection (CD4 count $\geq 350$ cells/mm3), acute HIV infection (CD4 count $<350$ cells $/ \mathrm{mm} 3$ who themselves were divided into sub-states according to breastfeeding, ART treatment and/or PMTCT intervention. The fundamental unit of time in the simulation was a month. Transition probabilities from one state to another were constant over time and based on observed data in Cameroon: the prevalence of HIV, access to antenatal care, coverage of maternal HIV testing, coverage of maternal CD4 cell count assessment and live birth rate. The MTCT HIV transmission probabilities depended on the timing of the mother's infection 
(prior/during pregnancy or lactation), CD4 cell count at different stages, the ART regimen and duration and the breastfeeding practices, thus the duration spent in each health state (see Additional file 1 for detailed calculations).

\section{Baseline input data}

Baseline inputs used to characterize our hypothetical cohort of pregnant and lactating women were derived from different data sources: current national surveys from National AIDS Control Committee and Cameroon's National Institute of Statistics [18, 19], clinical trials and cohort studies conducted in Cameroon or other resourcelimited settings in the absence of national data [20-23]. All data used are summarised in Table 1. We also assumed that HIV infection is associated with a lower fertility among HIV-infected women [24, 25]. The proportion of live births issued from an HIV-infected woman was estimated taking this fact into account, as well as the fact that woman may die during pregnancy.

\section{Model structure: calculation of mother-to-child transmission probabilities}

Mother-to-child-transmission (MTCT) of HIV can mainly occur during the second and third trimester of pregnancy, during delivery or breastfeeding [1]. Indeed, HIV transmission through breastfeeding has emerged as a substantial mode of MTCT among African breastfeeding populations and can occur in two different circumstances: among HIV prevalent mothers HIV-infected at delivery and among incident mothers HIV-infected while lactating. The risk of transmission through breastfeeding is cumulative according to the duration of breastfeeding and the longer the duration of breastfeeding, the greater the transmission risks [26-29]. Thus, we estimated three MTCT probabilities, using data from MTCT studies among pregnant and breastfeeding populations in Africa: 1/ the perinatal transmission probability at 6-week, 2/ the postnatal transmission probability and $3 /$ the postnatal transmission probability in those born to incident HIV-mothers who seroconverted while lactating. Additionally, we hypothesized that each of these three MTCT probabilities varied according to the maternal age group and CD4 count. Infants HIV status was computed using these estimated MTCT probabilities.

Details regarding the calculation of live births rate among HIV infected woman and the calculation of MTCT probabilities are available in an additional text file (see Additional file 1, which describes models used for the calculation of MTCT probabilities and live birth rate among HIV infected women).

Rates of mother-to-child transmission of HIV and population size of new paediatric HIV infections

Using our model we simulated 1, 000 cohorts of the population size of pregnant women expected in
Cameroon in 2011, through each state of the PMTCT cascade described in Fig. 1.

We calculated, using a Monte Carlo approach simulation, the MTCT rates including perinatal and postnatal transmission rates among prevalent HIV-infected mothers at birth and the postnatal transmission rate among incident mothers for these 1000 cohorts. We first modelled the probability for a mother to infect her infant, used that probability to estimate a number of HIV-infected infants born to mothers in the simulated cohort and divided that number by the number of children at risk of being HIVinfected in that cohort. Specifically, the final MTCT risks were calculated as follows: Perinatal transmission rate is equal to the average number of HIV-infected children at 6-weeks born alive to prevalent HIV-infected mothers divided by the total average number of live births from prevalent HIV-infected mothers. Postnatal transmission rate is equal to the average number of children HIVuninfected at 6-weeks who become infected beyond through breastfeeding, divided by the total average number of children born alive to prevalent and incident HIV-infected mothers. Postnatal transmission rate due to incident infection among lactating mothers: this rate is equal to the average number of breastfed HIV-infected children born alive to mothers HIV-uninfected at delivery but who seroconverted during breastfeeding, divided by the total average number of breastfed children born alive to incident HIV-infected mothers. Population size of new paediatric HIV infections is equal to the total average number of live born children from an HIV-infected prevalent mother at birth who become HIV-infected perinatally or during the breastfeeding period and the number of children HIV-infected through breastmilk from an incident HIV-infected lactating mother.

Finally, we examined the impact of different levels of uptake of PMTCT services on the MTCT rates and the number of new paediatric infections. Three scenarios were considered. First, we considered $100 \%$ uptake and with a $100 \%$ retention on treatment during the whole breastfeeding period. This meant that all pregnant women had access to antenatal care (ANC), HIV testing and counselling, disclosure of their HIV results; those who were infected had a CD4 assessment and were initiated on ART prophylaxis during pregnancy until the end of breastfeeding, as well for their child. In the second scenario, we considered a $90 \%$ uptake of PMTCT services, described as $100 \%$ access to ANC, $90 \%$ HIV testing and counselling, a $100 \%$ of HIV testing result disclosure and a $90 \%$ rate of ART prophylaxis during pregnancy. The third scenario meant $80 \%$ uptake of PMTCT services, described as $100 \%$ access to ANC, $80 \%$ HIV testing and counselling, a $100 \%$ of HIV testing result disclosure and an $80 \%$ rate of ART prophylaxis during pregnancy. 
Table 1 Pregnant women population groups and model key parameters according to the observed data in the different age groups

\begin{tabular}{|c|c|c|c|c|c|c|c|c|c|}
\hline Age groups (years) & $15-49$ & $15-19$ & $20-24$ & $25-29$ & $30-34$ & $35-39$ & $40-44$ & $45-49$ & Data source \\
\hline Total number of pregnant women expected & 995,533 & 231,982 & 202,093 & 173,223 & 125,437 & 108,513 & 80,638 & 73,647 & Cameroon $[18,19]$ \\
\hline HIV prevalence, $\%$ & 5.6 & 2.0 & 3.4 & 7.6 & 7.3 & 10.0 & 7.1 & 6.4 & Cameroon [18] \\
\hline Access to Antenatal Care), \% & 36.5 & & & & & & & & Cameroon [19] \\
\hline Prenatal HIV testing, \% & 29.3 & & & & & & & & Cameroon [19] \\
\hline Receipt of maternal HIV test result, \% & 92.4 & & & & & & & & Cameroon [19] \\
\hline Mortality during pregnancy, \% & 4 & & & & & & & & Cameroon [19] \\
\hline Live birth among HIV uninfected women, \% & 95 & & & & & & & & Cameroon $[9,19]$ \\
\hline Live birth among HIV infected women, \% & & 94.6 & 72.7 & 68.9 & 63.3 & 58.4 & 52.0 & 51.9 & {$[17,26]$} \\
\hline ARV prophylactic coverage for HIV infected pregnant women, \% & 20.3 & & & & & & & & Cameroon [19] \\
\hline CD4 cell count quantification for HIV infected women, \% & 6.9 & & & & & & & & Cameroon [19] \\
\hline Pregnant women ART eligible in ANC, \% & 60 & & & & & & & & Cameroon [19] \\
\hline ART coverage for eligible women in ANC, \% & 25 & & & & & & & & Cameroon [19] \\
\hline HIV MTCT rate at 6-week in the absence of MTCT, \% & 22 & & & & & & & & Sub-saharan Africa $[23,28]$ \\
\hline Treatment Effectiveness Expected & & & & & & & & & {$[21,23]$} \\
\hline -Short course ARV (\% reduction) & 86.7 & & & & & & & & \\
\hline -Long course ARV/ ART (\% reduction) & 93.7 & & & & & & & & \\
\hline $\begin{array}{l}\text { Rate of breastfeeding exposure among HIV exposed children known } \\
\text { at birth (\%) }\end{array}$ & 46 & & & & & & & & Cameroon, Pediacam $^{\mathrm{a}}$ 1, CIRCB field studies ${ }^{\mathrm{b}}$ \\
\hline $\begin{array}{l}\text { Rate of breastfeeding exposure among children not known to be HIV } \\
\text { exposed at birth, (\%) }\end{array}$ & 97 & & & & & & & & Cameron [18] \\
\hline $\begin{array}{l}\text { Intra-partum incidence of HIV infection (per } 100 \text { woman-year } \\
\text { of follow-up), \% }\end{array}$ & & 2 & 2 & 1.6 & 1.3 & 1 & 0.6 & 0.6 & Sub-saharan Africa [39-41] \\
\hline Incident HIV infections among lactating women, \% & 2.0 & & & & & & & & Zimbabwe $[27,31]$ \\
\hline Proportion of children still exposed to breastfeeding (age in months), \% & & & & & & & & & Cameroon [18] \\
\hline$[0,2[$ & 97.5 & & & & & & & & \\
\hline$[2,4[$ & 98.9 & & & & & & & & \\
\hline$[4,6[$ & 96.0 & & & & & & & & \\
\hline$[6,9[$ & 95.9 & & & & & & & & \\
\hline$[9,12[$ & 90.5 & & & & & & & & \\
\hline$[12,18[$ & 70.2 & & & & & & & & \\
\hline$[18,24]$ & 29.5 & & & & & & & & \\
\hline
\end{tabular}

Rate of exclusive breastfeeding among non HIV exposed children 
Table 1 Pregnant women population groups and model key parameters according to the observed data in the different age groups (Continued)

\begin{tabular}{ll}
\hline$[0,2[$ & 31.2 \\
{$[2,4[$} & 23.0 \\
{$[4,6[$} & 10.6 \\
{$[6,9[$} & 1.9 \\
{$[9,12[$} & 0.8 \\
{$[12,18[$} & 0.7 \\
{$[18,24]$} & 0.0
\end{tabular}

Rate of exclusive breastfeeding among HIV exposed children

Cameroon,

(age in months), \%

Pediacam $^{\mathrm{a}}$ 1, CIRCB field studies ${ }^{\mathrm{b}}$

\section{$[0,2[$}

86.6

$[2,6[$

$[6,12[$

$[12,18]$

Proportion of HIV exposed children who received postnatal ART, \%

$[6,12[$

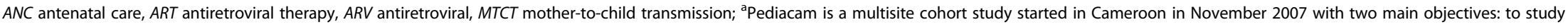
the feasibility and effectiveness, of early antiretroviral multi-therapy offered systematically to HIV-infected infants before 7 months of age; and to evaluate the humoral response of these children to vaccines of the Expanded Program of Immunization; ${ }^{b}$ Unpublished Early Infant Diagnosis of HIV data located at CIRCB 


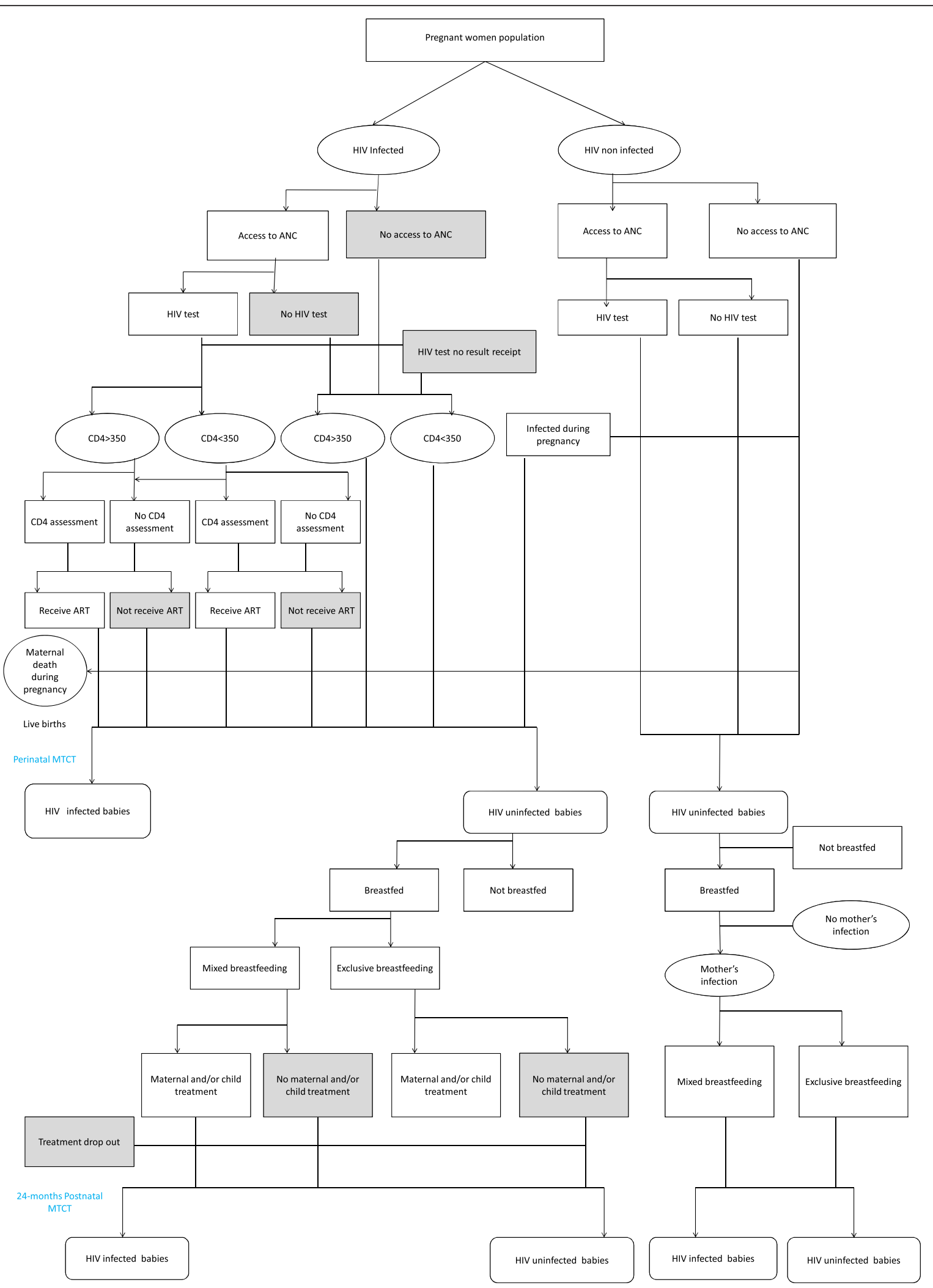

Fig. 1 PMTCT cascade. This figure shows the different state between pregnancy and delivery, then delivery and breastfeeding cessation, including PMTCT services offering: gray colour highlights the missed opportunities in the PMTCT process. Each oval represents a maternal health state, rounded rectangle represents child health state and rectangle represents clinical events and breastfeeding practices 
$95 \%$ confidence intervals of perinatal, postnatal and postnatal due to maternal incident infection rates, as well as the population size of new paediatric infections were derived from the 0.025 and 0.975 quintiles of 1000 subpopulation generated by bootstraps.

\section{Sensitivity analyses}

Sensitivity analyses of the results of the simulation model were conducted by varying in suitable intervals some key parameters in the prevention of MTCT of HIV, as described in Table 2. Sensitivity analyses were performed by varying these parameters in specific intervals based on the lowest and highest published values. For each key parameter 1000 values were randomly drew within the corresponding range. For each of these 1000 sets of randomly drawn parameter we simulated one cohort of the population size of pregnant women expected in Cameroon in 2011 and the associated MTCT rates were calculated.

In this work, all results were performed using $\mathrm{R}$ statistical software ( $R$ Core Team (2013). $R$ : A language and environment for statistical computing. $R$ Foundation for Statistical Computing, Vienna, Austria. URL http:// www.R-project.org/.).

\section{Results}

The simulation model was performed for the population of 995, 533 pregnant women expected in Cameroon in 2011 [19].

\section{Base-case analyses: fixed values of PMTCT cascade}

Available data showed a low rate of PMTCT services utilization (i.e. uptake of all components of the PMTCT cascade) everywhere in Cameroon, ranging from $6.9 \%$ to $36.5 \%$ in 2011 [19].

Table 3 presents the results of simulated mother-tochild transmission rates of HIV in both prevalent and incident HIV-infected mothers according to the observed coverage of PMTCT services in Cameroon in 2011. The overall MTCT rate, in both prevalent and incident HIV-infected mothers, was estimated to be $22.1 \%$ (95 \% Confidence Interval (CI): $18.6 \%-25.2 \%$ ) in 2011, resulting in 10, 403 new paediatric HIV infections (95\% CI: 9, 054-13, 345).

Estimates of the perinatal MTCT rate was $12.1 \%$ (95 \% CI: $8.1 \%-15.1 \%$ ), leading to 3, 758 new paediatric infections at 6-week of life (95\% CI: 3, 033-5, 077). During the postnatal period, the MTCT rate issued from prevalent HIV-infected women at delivery was $13.3 \%$ (95\% CI, $9.3 \%-17.8 \%$ ) corresponding to 3, 484 (95\% CI: $2,687-5,286)$ new paediatric infections. Among mothers who seroconverted during the breastfeeding period, the postnatal MTCT reached $20.8 \%$ (95 \% CI: $14.1 \%-26.9 \%$ ), corresponding to 3, 161 (95\% CI: 2, $337-4,679)$ new paediatric infections.

\section{Different PMTCT utilization coverage}

Table 4 presents the results of three simulated scenarios of MTCT rates of HIV and number of new infections in prevalent maternal population according to the observed coverage of PMTCT services in Cameroon in 2011in case of $100 \%$, median $90 \%$ and more likely of $80 \%$ uptake of PMTCT services.

When the model was run for a $100 \%$ level of consumption of PTMCT services among prevalent HIV pregnant women, we estimated the MTCT rate of HIV at 6-weeks to be $0.4 \%$ (95\% CI: $0.2 \%-1.1 \%)$, corresponding to 165 (95\% CI: 99-398) new paediatric perinatal infections. The postnatal transmission rate would be $0.8 \%$ (95 \% CI: $0.5 \%-1.6 \%$ ), corresponding to 149 (95 \% CI: 99-298) new paediatric postnatal infections. Thus, the global MTCT rate issued from prevalent HIV infected mothers would be $0.9 \%$ (95\% CI: $0.5 \%-1.7 \%$ ),

Table 2 Range of Model parameters for sensitivity analyses (results expressed in \%)

\begin{tabular}{|c|c|c|c|}
\hline Key parameters & Base case value & Range for sensitivity analyses & Data sources \\
\hline Access to ANC & 36.5 & $36-85$ & {$[18,19]$} \\
\hline HIV testing in ANC & 80.1 & $80-100$ & [19] \\
\hline ARV prophylactic coverage in ANC for HIV-infected women & 67 & $50-74$ & {$[2,19]$} \\
\hline CD4 cell count quantification for infected women in ANC & 22.9 & $22-100$ & [19] \\
\hline ART eligible in ANC & 60 & $40-60$ & [19] \\
\hline ART coverage for eligible women in ANC & 25 & $25-32$ & {$[3,19]$} \\
\hline Natural history of HIV MTCT at birth & 22 & $15-25$ & {$[23,28]$} \\
\hline Treatment Effectiveness & & & {$[21,23]$} \\
\hline -Short course ARV (reduction) & 86.7 & $86-95$ & \\
\hline -Long course ARV/ ART (reduction) & 93.7 & $93-95$ & \\
\hline Incidence rate among postpartum lactating women & 2.0 & $0.2-3.8$ & [31] \\
\hline
\end{tabular}

ANC antenatal care, $A R T$ antiretroviral therapy, ARV antiretroviral treatment, MTCT mother-to-child transmission 
Table 3 Simulated MTCT rates of HIV in prevalent and incident maternal population according to the observed coverage of PMTCT services in Cameroon in 2011

\begin{tabular}{llc}
\hline MTCT circumstances & $\begin{array}{l}\text { Estimated number of incident paediatric } \\
\text { infections [95\% Confidence Interval] }\end{array}$ & Estimated transmission rate (\%) \\
\hline Prevalent HIV-infected pregnant women & & $12.1[8.8-15.1]$ \\
Perinatal rate (<6 weeks of age) & $3,758[3,033-5,077]$ & $13.3[9.3-17.8]$ \\
Postnatal rate ( $>6$ weeks of age to 24 months) & $3,484[2,687-5,286]$ & $20.8[14.1-26.9]$ \\
Postnatal rate among incident maternal infection during breastfeeding & $3,161[2,337-4,679]$ & $22.1[18.6-25.2]$ \\
Total & $10,403[9,054-13,345]$ & 2 \\
\hline
\end{tabular}

MTCT mother-to-child transmission, PMTCT prevention of mother-to-child transmission

with a total number of new paediatric infections of 316 (95 \% CI: 198-631).

Assuming that PMTCT services were used at $90 \%$, the MTCT rate of HIV at 6-weeks would be $1.4 \%(95 \%$ CI: $0.5 \%-2.8 \%$ ), corresponding to 509 (95 \% CI: 199995) new paediatric perinatal infections, and the postnatal transmission rate would be $1.3 \%$ (95 \% CI: $0.5 \%-$ $3.0 \%$ ), corresponding to 205 (95\% CI: 99-497) new paediatric postnatal infections. In this scenario, the global MTCT rate issued from prevalent HIV-infected mothers would be $2.0 \%$ (95 \% CI: $0.9 \%-3.2 \%$ with a total of new paediatric infections reaching 711 (95\% CI: 306-1, 187).

When running the model for a $80 \%$ uptake of PTMCT services, the MTCT rate at 6-weeks would be $3.3 \%$ (95 \% CI: 1.3-5.6), corresponding to 1, 150 (95\% CI: 497-1, 891) new paediatric perinatal infections, and the postnatal transmission would be $2.1 \%$ (95\% CI: $0.6 \%-4.4 \%$ ) corresponding to 342 (95 \% CI: 99-696) new paediatric postnatal infections. In this scenario, the global MTCT rate issued from prevalent HIV infected mothers would $4.3 \%$ (95 \% CI: $2.4 \%-6.7 \%$ ), with a total number of new paediatric infections to be 1, 505 (95\% CI: 895-2, 354).

Hence, for prevalent HIV-infected mothers, reaching $100 \%$ uptake of PMTCT services would lead to a $96.9 \%$ (95\% CI: $93.9 \%-98.0 \%$ ) reduction of new paediatric infections in 2011. When PMTCT services are used at $90 \%$, the new paediatric infections rate would be reduce by $93.1 \%$ (95 \% CI: $88.5 \%-97.0 \%$ ) and by $85.5 \%$ (95\% CI: $77.3 \%-91.3 \%$ ) if PMTCT services are used at $80 \%$.

\section{Sensitivity analyses}

When varying identified key parameters in the model, the mean perinatal transmission rate was $10.6 \%$ (95\% CI: $6.2 \%-15.5 \%)$, the mean postnatal transmission rate among prevalent mothers was $12.3 \%$ (95 \% CI: $8.1 \%-$ $16.5 \%)$ and the mean postnatal transmission rate among incident HIV-infected lactating mothers was $20.1 \%$ (95\% CI, [11.3-29.5]). The population size of new

Table 4 Simulated scenarios of MTCT rates of HIV in prevalent maternal population according to the fixed coverage of PMTCT services in Cameroon in 2011

\begin{tabular}{|c|c|c|}
\hline \multicolumn{3}{|l|}{$100 \%$ of PMTCT services coverage } \\
\hline MTCT circumstances & $\begin{array}{l}\text { Estimated number of incident paediatric infections } \\
\text { [95\% Confidence Interval] }\end{array}$ & Estimated transmission rate (\%) \\
\hline Perinatal rate (<6 weeks of age) & 165 [99-398] & $0.4[0.2-1.1]$ \\
\hline Postnatal rate ( $>6$ weeks of age to 24 months) & 149 [99-298] & $0.8[0.5-1.6]$ \\
\hline Total & 316 [198-631] & $0.9[0.5-1.7]$ \\
\hline \multicolumn{3}{|l|}{$90 \%$ of PMTCT services coverage } \\
\hline Perinatal rate (<6 weeks of age) & 509 [199-995] & $1.4[0.5-2.8]$ \\
\hline Postnatal rate (> 6 weeks of age to 24 months) & 205 [99-497] & $1.3[0.5-3.0]$ \\
\hline Total & $711[306-1,187]$ & $2.0[0.9-3.2]$ \\
\hline \multicolumn{3}{|l|}{$80 \%$ of PMTCT services coverage } \\
\hline Perinatal rate ( $<6$ weeks of age) & $1,150[497-1,891]$ & $3.3[1.3-5.6]$ \\
\hline Postnatal rate (> 6 weeks of age to 24 months) & $342[99-696]$ & $2.1[0.6-4.4]$ \\
\hline Total & $1,505[895-2,354]$ & $4.3[2.4-6.7]$ \\
\hline
\end{tabular}

MTCT mother-to-child transmission PMTCT prevention of mother-to-child transmission 
paediatric infections in 2011 was 9, 131 cases $(95 \%$ CI: 4, 279-14, 834). Simulating improvements in coverage of prenatal services, prenatal HIV testing, CD4 cell count assessment among HIV-infected ARV for eligible women, PMTCT prophylactic services, improvement of treatment effectiveness, and the reduction of the incidence of HIV among lactating women led to the reduction of perinatal and postnatal transmission rates.

\section{Discussion}

We developed a discrete event simulation model with a stochastic structure to estimate the rates of MTCT of HIV and the number of new paediatric HIV infections in 2011 in Cameroon cumulating the two situations of prevalent HIV-infected mothers at delivery and incident HIV-infected mothers while lactating. First, despite the PMTCT services implementation, we still observed in 2011 an overall residual global MTCT rate that reached $22 \%$. Second, in this context of PMCT interventions, our results highlight that most of paediatric HIV infections occurred postnatally, through breastfeeding, and this was particularly high among the mothers who acquired HIV during lactation. Finally, in scenarios where the uptake of PMTCT is high i.e.100 \%, $90 \%$ and $80 \%$, we could reach significant reductions of MTCT by $96.6 \%, 93.1 \%$ and $85.5 \%$ respectively.

The results of this simulation model report an overall MTCT risk of $22 \%$ in 2011 which remains high and consistent with the $24 \%$ reported by the UNAIDS' Global Plan that same year $[7,30]$. We explain this high rate by a limited access to PMTCT interventions coupled with a low uptake of these services at different steps of the PMTCT cascade. Despite efforts towards eliminating new paediatric infections, Cameroon still faces limited availability of PMTCT services in primary health-care centres. In 2011, the country opted for the WHO Option A regimen for PMTCT prophylaxis. However, only $20 \%$ of identified HIV-infected pregnant women received a PMTCT prophylaxis [19], and $27 \%$ of their infants received a postnatal ART for prophylaxis [30]. It is urgent to expand the availability of PMTCT services within ANC and to improve the retention and access to services delivery over the whole MTCT period at risk, i.e. until the complete cessation of breastfeeding.

In our study, we also found that the risk of MTCT was highest in women who acquired HIV infection in the post-partum period. This result has already been reported in other settings [27]. Indeed, a study conducted in Zimbabwe showed that MTCT was 4.6 times higher in mothers who had seroconverted during breastfeeding compared to those who had been tested HIV-positive prior to delivery [27]. We explain this by a peak of maternal breast-milk viral load after the maternal HIV primo-infection [27, 31]. In our model, maternal infection during breastfeeding contributed significantly, for one-third, to the paediatric HIV burden. This result has been reported by other modelling studies, including one in South Africa where the proportion of MTCT was $26 \%$ in mothers who seroconverted after their first ANC visit [32, 33]. Another survey conducted in Mozambique, reported an MTCT rate in mothers who seroconverted during breastfeeding of $21 \%$ [34]. Nevertheless, despite a high HIV post-partum incidence in high prevalence settings in Africa, the HIV testing coverage in women in late pregnancy or in the postpartum period remains uncommon as testing practices often focus on the first ANC. In high HIV prevalence settings, early identification of incident infection in postpartum women and immediate testing of their exposed children is critical for initiating ART prophylaxis to prevent infection in infants and initiating early ART for those HIV-infected infants. Repeated HIV testing of HIV-uninfected mothers around delivery could allow identifying newly infected women and their exposed infants [35]. However, in resource-limited settings, the turnover period of these tests is often counted in weeks and the mother has already infected her child. In this context, it is primarily essential to focus on developing innovative primary HIV prevention programmes targeted on breastfeeding women and their partners [36]. Strategies to control postnatal MTCT should include community, behavioural and biomedical interventions focused to the vulnerable population of breastfeeding women.

According to the different PMTCT utilization rate scenarios analysed, we also demonstrated that the Global Plan target of reducing new paediatric infections by $90 \%$ could be achieved if all pregnant women used PMTCT services at each step of the PMTCT cascade. This result is consistent with another modelling study conducted using the Spectrum model, that reported a reduction of $79 \%$ in new paediatric infections in 25 countries, including Cameroon [37]. Decreasing markedly HIV MTCT transmission rates and eliminating new infections requires achieving family planning, primary HIV prevention of childbearing age women, and universal coverage of effective prenatal and postnatal antiretroviral regimen. In practice, Cameroon has achieved considerable progress, and in 2012, the ART coverage for PMTCT was estimated to be $64 \%$ [11]. Furthermore, Cameroon started implementing Option $\mathrm{B}+$ at the national level in 2014. Indeed, most of the efforts on PMTCT have been focused on improving access to prenatal antiretroviral prophylaxis. However, antiretroviral prophylaxis alone is insufficient and it is essential to improve the primary prevention and access to family planning. According to the Cameroon demographic health survey, $24 \%$ of women still have unmet needs for family planning [18]. Modelling studies have suggested that there will be significant reductions in the number of 
HIV-exposed infants if unmet needs are reduced [37]. Thus, it is essential to strengthen programmes that offer family planning services to both HIV-infected and uninfected pregnant women.

Methods for producing estimates have their limitations [38]. First, computer simulations simplify complex biological and operational processes and many assumptions involved some uncertainty. However, our results are consistent with those previously reported by other modelling studies, including the Spectrum model. Second, we used multiple-source data from different settings that were not all issued from Cameroon. This may have also impacted our assumptions in the estimation of transition probabilities between states. However, sensitivity analyses conducted found that the impact on estimates was minimal. In the other hand, our model don't take into account of maternal death during up to two months postpartum period. Consequently, the number of new paediatric HIV infections due to postnatal transmission could be overestimated in our model. Finally, in this analysis, we did not consider different PMTCT antiretroviral regimens. Indeed, it is still largely unclear which option is the most effective when implemented in a public healthcare system, but we felt that our average estimate of efficiency was likely [37]. The originality of this work is that most of the input PMTCT services and coverage components were issued from the Cameroon, which provides here a useful estimation of the paediatric infections projections for policymakers at the national level. The data presented here represent a base case scenario against which we will compare more recent estimates as they become available, reflecting other interventions as they are implemented, including option $\mathrm{B}+$ regimen for PMTCT prophylaxis.

\section{Conclusion}

Despite access to PMTCT services, paediatric HIV infections remain a concerning problem in Cameroon in 2011, with a high residual MTCT rate attributable to postnatal transmission. The national strategies planned to increase the PMTCT services coverage among HIV-exposed children, pregnant and lactating HIV-infected women to at least $90 \%$ for the 2011-2015 periods to substantially reduce new infant HIV infections. However, to reach the goal of the 'virtual elimination' of paediatric HIV, the national strategic plan needs to first fix ANC access and delivery of antenatal HIV testing to $100 \%$ for prevalent HIV-infected mother before delivery. An additional effort should be made to encourage pregnant women to use PMTCT services at all levels, at least until a complete breastfeeding cessation. Then, a substantial effort needs to be done to prevent HIV incidence in lactating women. This recurrent modelling informed by the national statistics will be helpful in guiding the national policy to PMTCT in Cameroon.

\section{Additional file}

Additional file 1: Methods of calculation of mother-to-child transmission probabilities and live births rate among HIV positive women. This additional file is a .docx file which contains description of mathematical models used to estimate mother-to-child transmission probabilities. It is also described here how live births rate among HIV positive women were calculated. (DOCX $37 \mathrm{~kb}$ )

\section{Abbreviations}

ANC: Antenatal Care; ART: Antiretroviral Therapy; CIRCB: Centre International de Référence Chantal Biya pour la recherche sur la prévention et la prise en charge du VIH/SIDA; MTCT: Mother To Child Transmission; PMTCT: Prevention of Mother To Child Transmission.

\section{Competing interests}

The authors have no competing interests to declare.

\section{Authors' contributions}

Model development: HLNN, HG, AA. Contributed to the coordination: $\mathrm{HG}, \mathrm{OOMO}-\mathrm{B}, \mathrm{AA}$. Conducted modelling analyses: HLNN, HG, AA. Critical interpretation of model results: HLNN, SD, VL, AA. Contributed the necessary data from their health facilities: CN, MT, PT, ID. Selected model input parameters: HLNN, $H G, S D, V L, A A$. Wrote the draft: HLNN, SD. Critical revision of the manuscript for important intellectual content: HLNN, SD, OOMO-B, CN, VL and AA. All authors read and approved the final manuscript.

\section{Acknowledgements}

The authors acknowledge Guy Severin Mahiané, PhD, Johns Hopkins University, Baltimore, USA, for comments on the model development, the National AIDS Control Committee (NACC) and the National Institute of Statistics of Cameroon.

\section{Funding}

Hermine L. Nguena Nguefack received funding from the French Embassy in Cameroon as a partial support for her PhD program, Sophie Desmonde is supported by the Sidaction Young Investigator Fellowship. The funders had no role in study design, data collection and analysis, decision to publish, or preparation of the manuscript.

\section{Author details}

${ }^{1}$ National Advanced School of Engineering, The University of Yaoundé I, PO Box 8390, Yaoundé, Cameroon. ${ }^{2}$ Inserm, U897 Bordeaux, France. ${ }^{3}$ Bordeaux School of Public Health, The University of Bordeaux, Bordeaux, France. ${ }^{4}$ Centre International de Référence Chantal Biya (CIRCB) pour la recherche sur la prévention et la prise en charge du VIH/SIDA, Yaoundé, Cameroun. ${ }^{5}$ Centre de Recherche Médicale et Sanitaire (CERMES), member of Réseau International des Instituts Pasteur, Niamey, Niger. ${ }^{6}$ Centre Pasteur du Cameroun, member of Réseau International des Instituts Pasteur, Yaoundé, Cameroun. ${ }^{7}$ Inserm U1027 Epidémiologie et analyses en santé publique: risques, maladies chroniques et handicaps, Université Paul Sabatier Toulouse 3, Toulouse, France.

Received: 21 July 2015 Accepted: 6 January 2016 Published online: 12 January 2016

\section{References}

1. De Cock KM, Fowler MG, Mercier E, De Vincenzi I, Saba J, Hoff E, et al. Prevention of mother-to-child hiv transmission in resource-poor countries: Translating research into policy and practice. JAMA. 2000;283(9):1175-82.

2. World Health Organisation, UNAIDS report on the global AIDS epidemic 2013. 2013. Available from: http://www.unaids.org/en/media/unaids/ contentassets/documents/epidemiology/2013/gr2013/UNAIDS_Global_ Report_2013_en.pdf. Accessed 19 Sept 2014.

3. World Health Organisation, UNAIDS Report on the global AIDS epidemic 2012. 2012 Available from: http://www.unaids.org/sites/default/files/media_ asset/20121120_UNAIDS_Global_Report_2012_with_annexes_en_1.pdf. Accessed 28 Nov 2015.

4. Leroy V, Ekouevi D, Becquet R, Viho I, Dequae-Merchadou L, Tonwe-Gold B. 18-month effectiveness of short-course antiretroviral regimens combined 
with alternatives to breastfeeding to prevent HIV mother-to-child transmission. PLOS ONE. 2008;3(2):e1645-e.

5. Tonwe-Gold B, Ekouevi DK, Viho I, Amani-Bosse C, Toure S, Coffie P, et al. Antiretroviral treatment and prevention of peripartum and postnatal HIV transmission in West Africa: evaluation of a two-tiered approach. PLoS Med. 2007;4(8):e257-e.

6. World Health Organisation, PMTCT strategic vision 2010-2015: preventing mother-to-child transmission of HIV to reach the UNGASS and Millennium Development Goals, 2010. 2010. Available from: http://www.who.int/hiv/ pub/mtct/strategic_vision.pdf, Accessed 28 Jan 2012

7. UNAIDS, Global Plan towards the elimination of new HIV infections among children by 2015 and keeping their mothers alive. 2011. Available from: http://www.unaids.org/sites/default/files/media_asset/20110609_JC2137_ Global-Plan-Elimination-HIV-Children_en_1.pdf. Accessed 28 Nov 2015.

8. World Health Organisation, Antiretroviral Therapy for HIV infection in infants and children: Towards universal access, Recommendations for a public health approach, 2010 revision, WHO Library Cataloguing-in-Publication Data. 2010. ISBN 9789241599801.

9. MINSANTE-CNLS Cameroun. Rapport national de suivi de la déclaration politique sur le VIH/SIDA au Cameroun, Rapport national, 2012. Available http://www. unaids.org/sites/default/files/en/dataanalysis/knowyourresponse/countryprogress reports/2012countries/ce_CM_Narrative_Report.pdf. Accessed 08 Jan 2016.

10. Penazzato $M$, Bendaud V, Nelson L, Stover J, Mahy M. Estimating future trends in paediatric HIV. AIDS. 2014;28 Suppl 4:S445-51.

11. UNAIDS, Progress report on the global plan towards the elimination of new HIV infections among children by 2015 and keeping their mothers alive. 2013. Available from: http://www.unaids.org/sites/default/files/en/media/ unaids/contentassets/documents/unaidspublication/2013/20130625_ progress_global_plan_en.pdf. Accessed 5 Mar 2014.

12. Tudor Car L, Van Velthoven MH, Brusamento S, Elmoniry H, Car J, Majeed A, et al. Integrating prevention of mother-to-child HIV transmission programs to improve uptake: a systematic review. PLoS ONE. 2012;7(4):e35268.

13. Vieira IT, Harper PR, Shahani AK, De Senna V. Mother-to-child transmission of HIV: a simulation-based approach for the evaluation of intervention strategies. J Oper Res Soc. 2003;54:713-22. doi:10.1057/palgrave.jors.2601566.

14. Ciaranello AL, Perez F, Maruva M, Chu J, Engelsmann B, Keatinge J, et al. WHO 2010 Guidelines for Prevention of Mother-to-Child HIV Transmission in Zimbabwe: Modeling Clinical Outcomes in Infants and Mothers. PLoS ONE. 2011;6(6):e20224. doi:10.1371/journal.pone.0020224.

15. Stover J, Brown T, Marston M. Updates to the Spectrum/Estimation and Projection Package (EPP) model to estimate HIV trends for adults and children. Sex Transm Infect. 2012;88:11 1i16. doi:10.1136/sextrans2012-050640.

16. Stover J, Johnson P, Hallett T, Marston M, Becquet R, Timaeus M. The Spectrum projection package: improvements in estimating incidence by age and sex, mother-to-child transmission, HIV progression in children and double orphans. Sex Transm Infect. 2010;86 Suppl 2:i16-21. doi:10.1136/sti.2010.044222.

17. Bollinger L, Stover J, Cooper-Arnold K, PMTCT Version1: A Decision Model to evaluate strategies to prevent mother-to-child transmission of HIV. Spectrum System of Policy models, 2002. Available: http://www.futuresgroup.com/files/ softwaremodels/PMTCTmnE.pdf. Accessed 08 Jan 2016.

18. Institut Nationale de la Statistique du Cameroun. Enquête Démographique et de Santé et à Indicateurs Multiples (EDS-MICS) 2011. Sep-2012. Available from: http://dhsprogram.com/pubs/pdf/FR260/FR260.pdf

19. Comité National de Lutte Contre le SIDA du Cameroun. Vers l'élimination virtuelle de la transmission du VIH de la mère à l'enfant à I'horizon 2015, rapport de progrès numéro 6, année 2011', Rapport de progrès, 2012 Available: ftp://www.cnls.cm/docs/publications/rapports_annuels/Rapport_ Progr\%C3\%83\%C2\%A8s_PTME_N6.pdf. Accessed 08 Jan 2016.

20. World Health Organisation, Towards universal access: scaling up priority HIV/ AIDS interventions in the health sector: progress report 2010. France, 2010. Available: http://apps.who.int/iris/bitstream/10665/44443/1/9789241500395_ eng.pdf. Accessed 08 Jan 2016.

21. Joint United Nations Programme on HIV/AIDS and World Health Organisation, HIV in Pregnancy: A Review. 1998. Available: http://www. unaids.org/sites/default/files/media_asset/jc151-hiv-in-pregnancy_en_1.pdf. Accessed 08 Jan 2016

22. World Health Organisation, Guide pour la mise l'échelle au plan mondial de la prévention de la transmission mère-enfant du VIH: vers un accès universel pour les femmes, les nourrissons et les jeunes enfants et pour l'élimination du VIH et du SIDA. 2007. Available: http://www.who.int/hiv/ mtct/PMTCT_frWEBNov26.pdf. Accessed 08 Jan 2016.
23. Rollins N, Mahy M, Becquet R, Khun L, Creek T, Mofenson L. Estimates of peripartum and postnatal mother-to-child transmission probabilities of HIV for use in Spectrum and other population-based models. Sex Transm Infect. 2012;88:i44-51. doi:10.1136/sextrans-2012-0580709.

24. Loko MA, Toure S, Dakoury-Dogbo N, Gabillard D, Leroy $\vee$ and Anglaret X. Decreasing incidence of pregnancy by decreasing CD4 cell count in HIVinfected women in Cote d'Ivoire: a 7-year cohort study. AIDS. 2005;19(4):443-5.

25. Gregson S, Zaba B, Hunter S. The impact of HIV-1 on fertility in sub-saharan Africa: causes and consequences. Available from: http://www.un.org/en/ development/desa/population/events/pdf/expert/4/zaba.pdf. Accessed 2014 January 17.

26. Chen WJ, Walker N. Fertility of HIV-infected women: insights from Demographic and Health Surveys. Sex Transm Infect. 2010;86 Suppl 2:ii22-7.

27. Humphrey JH, Marinda E, Mutasa K, Moulton L, lliff P, Ntozini R, et al. Mother to child transmission of HIV among Zimbabwean women who seroconverted postnatally: prospective cohort study. BMJ. 2010;341:c6580.

28. World Health Organisation, HIV transmission through breastfeeding : a review of available evidence: 2007 update. 2008. Available from: http:// apps.who.int/iris/bitstream/10665/43879/1/9789241596596_eng.pdf. Accessed 08 Jan 2016.

29. Leroy V, Newell M, Dabis F, Peckham C, Van de Perre P, for the Ghent International Working Group on Mother-to-Child Transmission of HIV. Late postnatal Mother-to-Child transmission of HIV-1 infection: author's reply. Lancet. 1998;352:1630.

30. UNAIDS, Together we will end AIDS 2012. 2012 Available from: http://www. unaids.org/sites/default/files/en/media/unaids/contentassets/documents/ epidemiology/2012/JC2296_UNAIDS_TogetherReport_2012_en.pdf. Accessed 28 Nov 2015.

31. Drake AL, Wagner A, Richardson B, John-Stewart G. Incident HIV during Pregnancy and Postpartum and Risk of Mother-to-Child HIV Transmission: A Systematic Review and Meta-Analysis. PLoS Med. 2014;11(2):e1001608. doi:10.1371/journal.pmed.1001608.

32. Warren CE, Abuya T, Askew I. Family planning practices and pregnancy intentions among HIV-positive and HIV-negative postpartum women in Swaziland: a cross sectional survey, BMC Pregnancy Childbirth 2013;13:150. Epub 17/07/2013.

33. Van de Perre $P$, Simonon A, Msellati $P$, Hitimana DG, Vaira D, Bazubagira A, et al. Postnatal transmission of Human Immunodeficiency Virus type-1 from mother to infant. A prospective cohort study in Kigali, Rwanda. N Engl J Med. 1991;325:593-8.

34. De Schacht C, Mabunda N, Ferreira OC, Ismael N, Calu N, Santos I, et al. High HIV incidence in the postpartum period sustains vertical transmission in settings with generalized epidemics: a cohort study in Southern Mozambique. J Int AIDS Soc. 2014;17:18808. Epub 19/03/2014.

35. Moodley D, Esterhuizen TM, Pather T, Chetty V, Ngaleka L. High HIV incidence during pregnancy: compelling reason for repeat HIV testing. AIDS. 2009;23(10):1255-9. Epub 21/05/2009.

36. Leroy $V$, Van de Perre $P$, Lepage $P$, Saba J, Nsengumuremyi F, Simonon A, et al. Seroincidence of HIV-1 infection in African women of reproductive age: a prospective cohort study in Kigali, Rwanda, 1988-1992. AIDS. 1994;8(7):983-6.

37. Mahy M, Stover J, Kiragu K, Hayashi C, Akwara P, Luo C, et al. What will it take to achieve virtual elimination of mother-to-child transmission of HIV? An assessment of current progress and future needs. Sex Transm Infect. 2010;86 Suppl 2:ii48-55.

38. Hallett T, Zaba B, Stover J, Brown T, Slaymaker E, Gregson S, et al. Embracing different approaches to estimating HIV incidence, prevalence and mortality'. AIDS. 2014;28 Suppl 4:S523-32.

39. Dunn DT, Newell ML, Ades AE, Peckham CS. Risk of human immunodeficiency virus type 1 transmission through breastfeeding. Lancet. 1992;340:585-8.

40. Humphrey JH, Hargrove JW, Malaba LC, lliff PJ, Moulton LH, Mutasa K, et al. HIV incidence among post-partum women in Zimbabwe: risk actors and the effect of vitamin A supplementation. AIDS. 2006;20:1437-46.

41. The HIV Modelling Consortium Treatment as Prevention Editorial Writing Group. HIV Treatment as Prevention: Models, Data, and Questions Towards Evidence-Based Decision-Making. PLoS Med. 2012;9(7):e1001259. doi:10.1371/journal.pmed.1001259.

42. Rosen S, Fox MP, Gill CJ. Patient retention in antiretroviral therapy programs in sub-Saharan Africa: A systematic review. PLoS Med. 2007;4(10):e298. doi:10.1371/journal.pmed.0040298. 